\title{
Acknowledgement of Reviewers for 2019
}

\section{Vassilios A. Tsihrintzis ${ }^{1}$}

Published online: 8 February 2020

(C) Springer Nature Switzerland AG 2020

The Editor-in-Chief and Publisher of Environmental Processes acknowledge the colleagues listed below who provided excellent reviews of papers submitted to the journal during the period January 1, 2019 to December 31, 2019.

Moussa Abbas

Syamsul Rizal Abd Shukor

Hany Abdel Ghafar

Mohamed Abdel Salam

Azza M. Abdel-Aty

Ibrahim Abdelfattah

Konstantinos Abeliotis

Ozeair Abessi

Marina Aboal

Ahmed A. Abokifa

Ferenc Ács

Vicenc Acuna

Subhas Adak

Werner Aeschbach-Hertig

Rajat Agarwal

Aqeel Ahmad

Noordin B. Ahmad

Christos Akratos

Rida Al-Adamat

Furat Al-Faraj

A.A. Al-Gheethi

Tadesse Alemu

Hazrat Ali
Mumtaz Ali

Mohammad N. Almasri

Javier Almorox

N. D. Ananyeva

M. C. Anderson

Leonardo Andrade

Panagiotis Angelidis

Robert Annear

V.Z. Antonopoulos

Georgia Antonopoulou

Jesus Manuel Anzano

David Aot

P.A. Azeez

Alireza Baghban

Chengke Bai

Guru Balamuguran

Jose M Baldasano

Carluvy Baptista-Salazar

Georgios Bariamis

Rosario Barranquero

Bartosz Bartkowski

Alica Bartosova

M.A. Behnajady
Miray Bekbolet

Vasilis Bellos

Christophe Bengoa

Marc Berenguer

Elizabeth A. Bergey

Mita Bhattacharya

Nishan Bhattarai

Rabin Bhattarai

Haq Nawaz Bhatti

Chandrashekhar Bhuiyan

Stefan Bilașco

Dimitra Bobori

Jean-Claude Bollinger

Fabian A. Bombardelli

Charles Bong

Boris Bonn

Lukasz Borek

Edward Bormashenko

Iosif Botetzagias

James Bowen

Adina Bragaru

Svetlana Bratkova

Jonathan Bray

Vassilios A. Tsihrintzis

tsihrin@otenet.gr; tsihrin@survey.ntua.gr

1 Centre for the Assessment of Natural Hazards and Proactive Planning \& Laboratory of Reclamation Works and Water Resources Management, National Technical University of Athens, Greece 


\begin{tabular}{|c|c|c|}
\hline Ilija Brceski & Samy El Mahdy & Eko Handayanto \\
\hline John Buchanan & Luis G. Elsitdié & Oliver Happel \\
\hline Tommaso Caloiero & Mohamed Enan & Saeedeh Hashemian \\
\hline Massimo Capobianco & Carlos Esse & Zulkarnain Hassan \\
\hline Rafael Carmona-Benítez & Ana Estrada & Artin Hatzikioseyian \\
\hline Jose Carrillo & Vasilis Evagelopoulos & Yolanda S. Hedberg \\
\hline Vincent Chaplot & Massimiliano Fabbricino & Salim Heddam \\
\hline Somsubhra Chattopadhyay & Hui Fan & Oliver Hensengerth \\
\hline Ghanshyam Chauhan & Junliang Fan & Mark Hernandez \\
\hline Wenshing Chen & Lu Fan & Edgar Hiller \\
\hline Yudao Chen & Agata Fargasova & Yuh-Shan Ho \\
\hline Ming-Hsun Cheng & Stefano Federico & Seyed-Mohammad Hosseini- \\
\hline Po-Kuan Chiang & Leticia Andrea Fernandez & Moghari \\
\hline Kangwoo Cho & J. Fernández-Pato & V. Hrissanthou \\
\hline G.C. Christodoulou & Dolores Fidelibus & Janice Hudson \\
\hline Chee Yap Chung & Manuel Figueredo & Muharrem Ince \\
\hline Filip Ciesielczyk & Jose Font & Che Fauziah Ishak \\
\hline Diana Cocârță & K.Y. Foo & Muhammad Ishaq \\
\hline F. Colangelo & Simona Fratianni & S. Ismadji \\
\hline Ian Colbeck & Chuck Frihart & Sharad Jain \\
\hline M.C. Costa & Zacharias Frontistis & Yefang Jiang \\
\hline Abumalé Cruz-Salomón & Vinay Kumar Gaddam & Jennifer Jones \\
\hline Enshirah Da'na & Chen Gang & Ganesh Joshi \\
\hline Bahareh Dabirmanesh & Guangyao Gao & Damir Jukic \\
\hline Dominika Dabrowska & Naiyun Gao & Dimitrios Kaimaris \\
\hline Filiz Dadaser-Celik & Celso Garcia & Ajay S. Kalamdhad \\
\hline Christos Damalas & Sergi Garcia-Segura & Dayanand Kalyani \\
\hline Eric Daniels & Rubia Zahid Gaur & Vassilis Kanakoudis \\
\hline Nguyen-Khoi Dao & Seyyed Hossein Ghoreishi & Andreas Kandelbauer \\
\hline Mohammad Darand & Najafabadi & Mitthan Kansal \\
\hline Shubhabrata Datta & Georgios Gikas & A.R. Karbassi \\
\hline Ralph K. Davis & Eric Gilleland & Janhavi M. Karekar \\
\hline Les Dawes & A.K. Giri & Efthimios Karymbalis \\
\hline Mohammad Hadi Dehghani & C.R. Girish & Harsimranpreet Kaur \\
\hline Alebachew Demoz & Kun-Yi Goel & Rijan Bhakta Kayastha \\
\hline Jin-Pei Deng & S.I.L. Gomes & Mohd Yawar Khan \\
\hline N. Dercas & E. González & Vinita Khandegar \\
\hline Georgios Deskos & Girish Gopinath & Medhi Khashei \\
\hline Ivan Deviatkin & Kadarkarai Govindan & Maryam Khozaei \\
\hline Cristina Di Salvo & Skok Gregor & Sang Jae Kim \\
\hline Vasileios Diamantis & Ester Guaus & Ichiro Kimura \\
\hline Isabel Diaz & Ali Gul & Vasileios Kitsikoudis \\
\hline Anatoli Dimoglo & Mohammad Shahid Gulgundi & Yury Koksharov \\
\hline Lifeng Ding & Rishabh Gupta & Dimitrios Komilis \\
\hline Eyup Dogan & Ahmet Gürses & Ioannis Kourtis \\
\hline Eva Domingues & Chris M. Hall & Apurav Krishna Koyande \\
\hline Hefni Effendi & Nasrul Hamidin & Khand Kul \\
\hline
\end{tabular}




\begin{tabular}{|c|c|c|}
\hline Aditi Kulshrestha & Paraschos Melidis & José A. Pellicer \\
\hline P. Senthil Kumar & Carlos Mello & Carlo Perego \\
\hline Preeti Kumari & Yannis Mertzanidis & Christos Petalas \\
\hline Yaswanth Kuthati & Bogdan Mihai & Christodoulos Pilinis \\
\hline Haim Kutiel & László Miklós & F. Pliakas \\
\hline M. Kwiatkowski & Elham Mirrasooli & Senthil Kumar Ponnusamy \\
\hline Bo Lai & M. Monirul Qader Mirza & Cristina Postigo \\
\hline Hugo Lambert & Akhilesh Mishra & K. Poulios \\
\hline Nikolaos Lambrakis & Dhirendra Mishra & Taotao Qiang \\
\hline Yasir Latif & N.K. Mondal & Botao Qin \\
\hline Lecheng Lei & Massimo Moretti & Xiaoqing Qiu \\
\hline Nuno Carlos Leitão & Rogeiro Mota & Margarida Quina \\
\hline David L. Lewis & Abdelmajid Moumen & Emad Radwan \\
\hline Huashou Li & Konstantinos Moutsopoulos & Mohammad Rahman \\
\hline Jianzhu Li & Mohammed Umar Mustapha & Raja Shazrin Shah Raja \\
\hline Peiyue Li & Mehmet Mete Mutlu & Ehsan Shah \\
\hline Kang Liang & I. Nalbantis & AL. Ramanathan \\
\hline Liqiao Liang & Ahmed Nasr & Vanessa Ramos \\
\hline Brantley Liddle & Mu Naushad & Kabir Rasouli \\
\hline Kun-Yi Andrew Lin & Chuck Chuan Ng & Rita Razauskaite \\
\hline Maozi Lin & Jafar Nouri & Edna Ríos-Valdovinos \\
\hline De Li Liu & Constantinos Noutsopoulos & Paula Rodriguez-Escales \\
\hline Xianfeng Liu & Spyridon Ntougias & Jörg Römbke \\
\hline Hugo Loaiciga & A. Oliveira & Roberto Rosal \\
\hline Antonia Longobardi & Sukru Omur & E. Rosales \\
\hline Athanasios Loukas & Chukwudi O. Onwosi & Klara Rusevova Crincoli \\
\hline Ming-Chun Lu & Donde Oscar & Kathryn Russell \\
\hline Yongjun Lu & Hook Osri & Karen R. Ryberg \\
\hline Evdoxia Lykoudi & Ayla Özer & Mir Jafar Sadegh Safari \\
\hline C. A. Madera-Parra & Siniša Ozimec & Masahiro Sakata \\
\hline P. Mageshkumar & Ilhan Ozturk & Michael Sakellariou \\
\hline Ratan Kumar Majumder & S. Pagliara & Kazuyuki Sakuma \\
\hline Dimitris Makris & Michele Palermo & S. Samson \\
\hline Giorgos Mallinis & Yiannis Panagopoulos & Lucas Santos-Juanes \\
\hline Ioannis Manariotis & Rames Panda & Trailokya Saud \\
\hline Sachin A. Mandavgane & Rajendra Prasad Pandey & Simona Schwarz \\
\hline Ciro Manzo & Basil Papadopoulos & Jan Seibert \\
\hline Michael D. Marsolek & Maria Papadopoulou & William R. Selbig \\
\hline Eduardo Marson & Vassiliki Papaevangelou & N. Selvaraju \\
\hline Pau Marti & Ino Papageorgaki & Vasanthi Sethu \\
\hline Fabrice Martin-Laurent & Ilias Papakonstantis & Amin Shaban \\
\hline Josep Mas-Pla & Panayotis Papanikolaou & Sameer Shadeed \\
\hline Ilyas Masih & G. Papaschinopoulos & Vijay Shankar \\
\hline M. Masood & Angeliki Paraskevopoulou & Jinyou Shen \\
\hline David Massey & Petros Patias & P. F. Sheridan \\
\hline Micòl Mastrocicco & Georgios Pavlikakis & Sohleila Shokrollahzadeh \\
\hline Sina Matavos-Aramyan & Haiyan Pei & Amna Rehana Siddiqui \\
\hline
\end{tabular}




$\begin{array}{lll}\text { Vasil D. Simeonov } & \text { Dimitris Tigkas } & \text { Jingjing Xie } \\ \text { Laishram Singh } & \text { Keesari Tirumalesh } & \text { Kaizhi Xie } \\ \text { P. Singh } & \text { K. Tokmakidis } & \text { Zongqiang Xie } \\ \text { Rajesh Singh } & \text { Konstantia Tolika } & \text { Jiang Xu } \\ \text { Santosh Kumar Singh } & \text { Fernando Toyohiko Wakida } & \text { Linyu Xu } \\ \text { Umesh K. Singh } & \text { Petros Tsakiridis } & \text { Baolu Yang } \\ \text { Waikhom Singh } & \text { Vassilios Tsihrintzis } & \text { Panayotis Yannopoulos } \\ \text { Maros Sirotiak } & \text { Stavroula Tsitsifli } & \text { Aijun Yao } \\ \text { Charalampos Skoulikaris } & \text { Dimitra Vagiona } & \text { Chee Kong Yap } \\ \text { S. Sobhanardakani } & \text { John T. Van Stan } & \text { Zaher Yaseen } \\ \text { Ioannis Sofos } & \text { I. Varsányi } & \text { Zaher Mundher Yaseen } \\ \text { Sait Sofuoglu } & \text { Perola de Castro Vasconcellos } & \text { Ulku Yetis } \\ \text { Maros Soldan } & \text { Arya Vazirzadeh } & \text { Daqiang Yin } \\ \text { Naglaa Farag Soliman } & \text { Danae Venieri } & \text { Naoto Yoshida } \\ \text { Manuel Soto } & \text { K. Voudouris } & \text { Jan Yperman } \\ \text { S. A. Spencer } & \text { Evangelos Voudrias } & \text { Jiaguo Yu } \\ \text { Mike Spiliotis } & \text { Zisis Vryzas } & \text { Y. Yu } \\ \text { Venkataramana Sridhar } & \text { Jinquan Wan } & \text { Abhijit Zende } \\ \text { Alexandros Stefanakis } & \text { Dongbo Wang } & \text { Qingming Zhang } \\ \text { Baolin Su } & \text { Fenglong Wang } & \text { Ya-feng Zhang } \\ \text { Daniel O Suman } & \text { Qiuxia Wang } & \text { Yulong Zhang } \\ \text { Bai-Ye Sun } & \text { Xiuguo Wang } & \text { Junbin Zhao } \\ \text { Georgios Sylaios } & \text { Mark A. Widdowson } & \text { Binghui Zheng } \\ \text { Adnan Tahir } & \text { A. Wierschem } & \text { Liugen Zheng } \\ \text { V. Tarabara } & \text { John Wilson } & \text { Hussein Zolgharmein } \\ \text { G. Tartari } & \text { Małgorzata Wojtkowska } & \text { Antonis Zorpas } \\ \text { Athanasia G Tekerlekopoulou } & \text { Fengzhi Wu } & \text { Ioanna Zotou } \\ \text { Santosh Thampi } & \text { Jinglu Wu } & \\ \text { N. Theodossiou } & \text { L.F. Wu } & \\ & & \end{array}$

Publisher's Note Springer Nature remains neutral with regard to jurisdictional claims in published maps and institutional affiliations. 